\title{
Vision Analysis in Detecting Abnormal Breathing Activity in application to Diagnosis of Obstructive Sleep Apnoea
}

\author{
Ching Wei Wang, Amr Ahmed and Andrew Hunter \\ Vision and Artificial Intelligence Group, Department of Computing \& Informatics, University of Lincoln \\ Brayford Pool, Lincoln LN6 7TS, United Kingdom \\ \{cweiwang | aahmed | ahunter\}@lincoln.ac.uk
}

\begin{abstract}
Recognizing abnormal breathing activity from body movement is a challenging task in machine vision. In this paper, we present a non-intrusive automatic video monitoring technique for detecting abnormal breathing activities and assisting in diagnosis of obstructive sleep apnoea. The proposed technique utilizes infrared video information and avoids imposing geometric or positional constraints on the patient. The technique also deals with fully or partially obscured patients' body. A continuously updated breathing activity template is built for distinguishing general body movement from breathing behavior.
\end{abstract}

Index Terms - breath monitoring, behavior recognition, vision analysis, respiration monitoring

\section{INTRODUCTION}

Obstructive Sleep Apnoea Syndrome is increasingly recognised as a common and serious condition, leading to sleep disturbance and consequential daytime sleepiness. Apnoea refers to short spells when breathing stops. In obstructive sleep apnoea, the throat constricts during sleep, preventing breathing; the apnoea episode often ends with a loud snore and/or gasp. The event is sufficient to open the throat muscles to allow breathing, and the patient usually falls asleep again so quickly that the event is not remembered. In obstructive sleep apnoea, this cycle repeats itself throughout the night as the muscles relax and the throat blocks again. The frequency of the episode determines the severity of the syndrome [12]. Hence, a further investigation on monitoring human breathing activity is needed for diagnosis of sleep disorders.

Current breathing monitoring techniques can be categorized into two types: invasive and non-invasive. The invasive type includes techniques such as using a belt [1] or strap [2] to track changes in body circumference during the respiratory cycle, stick-on electrodes as a heart-respiratory monitor [3, 4], and a nasal temperature probe [9]. Noninvasive types include techniques such as using audio analysis to monitor tidal volumes from human breathing activity [5], a vibration sensor [6,7], and thermal imaging $[10,11]$.

Theses approaches have various limitations in application to diagnosis of sleep disorders. The obtrusive nature of invasive monitoring equipments can disturb sleep and therefore compromise results. Although thermistors sense differences in temperature, they do not have a linear relationship with true airflow. In other words, the thermistors may not be sensitive for detecting hypopneas [8]. Nasal pressure has a linear approximation of airflow but can produce false-positive events and low quality signals if patients are mouth breathing [8]. In strap systems, if the tension on the strap is not calibrated the system will not track the respiration motion correctly, so that adjustment may be necessary. Also, measurements on patients with shallow and abdominal breathing patterns may fail because the sensor cannot track adequately in a reproducible manner if the chest displacements during normal breathing and breath-hold are not distinctly different. Regarding thermal imaging, there are position limitations and geometric constraints for targeting faces $[10,11]$.

In this paper, we develop a non-intrusive monitoring technique without geometric constraints and position limitations, using infrared video information, for identifying abnormal breathing activity in application to diagnosis of obstructive sleep apnoea. Shallow and abdominal breathing patterns do not affect the performance of the proposed approach. A continuously updated 2D breathing activity template, the shape of general breath movement, is built in order to recognize breathing behavior.

The outline of this paper is as follows. In section 2 we analyze human breathing behavior and present the concepts and algorithms of the proposed approach. The experimental setup and results are illustrated in section 3 and 4 . The conclusion and future work are described in section 5 .

\section{METHOD}

\section{A. Objectives}

The aim of our research is to support the diagnosis of obstructive sleep apnoea. Our objective is (1) to detect abnormal breathing episode. This requires us also (2) to distinguish breathing movements from general body movements.

\section{B. Technical Analysis in Breathing Behavior}

We analyze human breathing behavior and compare it with general body movement. Two important features of breathing activity were observed as described below.

1) The elements of the entire surface move forward and backward approximately to their previous position: In contrast, during general body movement the elements move to a different position. 


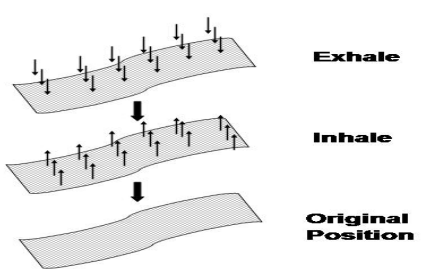

Fig. 1 Elements Moving Flow in a Breath Cycle

2) Breathing is a relatively slow motion activity: The movement is relatively slow, compared with general body movement. Hence, in order to detect breathing activity, we need to compare frames in larger intervals.

\section{Design}

1) Shapes of Movement: Discover the layout of movements, which are the differences between current frame and the constructed background frame.

2) Degree of Motion: The total number of data points that are different between current frame and background.

3) Adaptive Background Model: The model is designed as a combination of many frames for (1) memorizing old scenes and determining if the surface moves back to previous position and for (2) detecting the breathing movements, which are in slow motion mode.

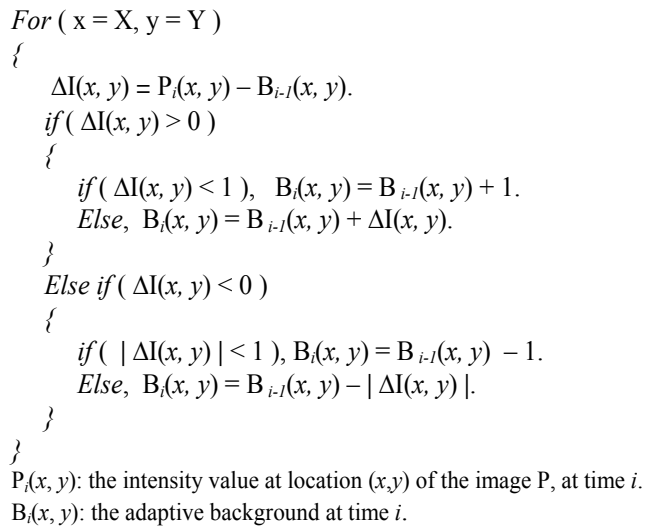

Fig. 2 Adaptive Background Model

The adaptive background model is crucial for distinguishing general body movement and breathing movement. Fig. 3 demonstrates that the duration for breathing movements is relatively small $\left(\mathrm{D}_{3}<\mathrm{D}_{2}\right)$ because the elements of the surface move back to the previous location.

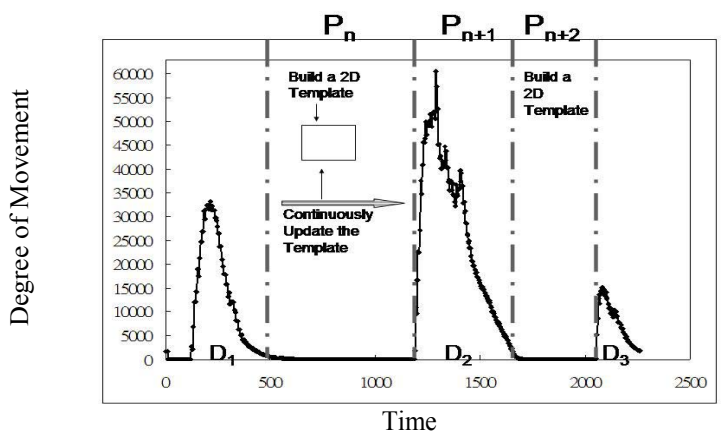

Fig. 3 Overall Procedure in Temporal Space
4) Continuously Updated $2 D$ Breath Activity Template: While the body is in general breathing mode, a 2D breath activity template is built and continuously updated over time. The template represents the shape of the general breathing movement. It is utilized to distinguish body movements from abnormal breath activities (See Fig. 4).

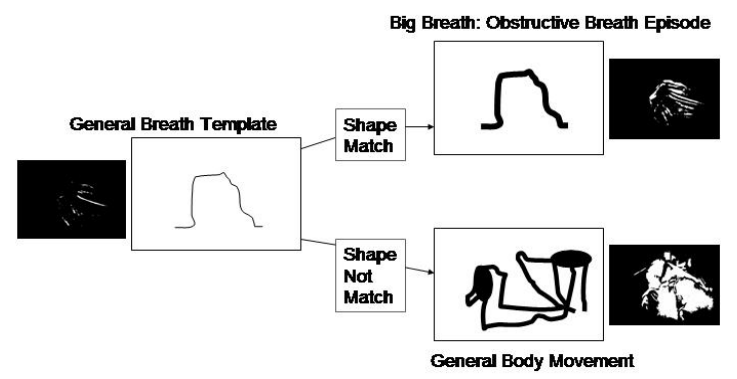

Fig. 4 Template Matching

Fig. 3 presents the overall procedure in temporal space. In $\mathrm{P}_{\mathrm{n}}$, the status is in general breathing mode, and during that period the system will continuously develop the breath activity template, which is further used for template matching in $\mathrm{P}_{\mathrm{n}+1}$ in order to determine if the movement is breathing behavior or not. In $\mathrm{P}_{\mathrm{n}+2}$, a new 2D template will be built over again. Fig. 5 illustrates analysis results from one experimental result.

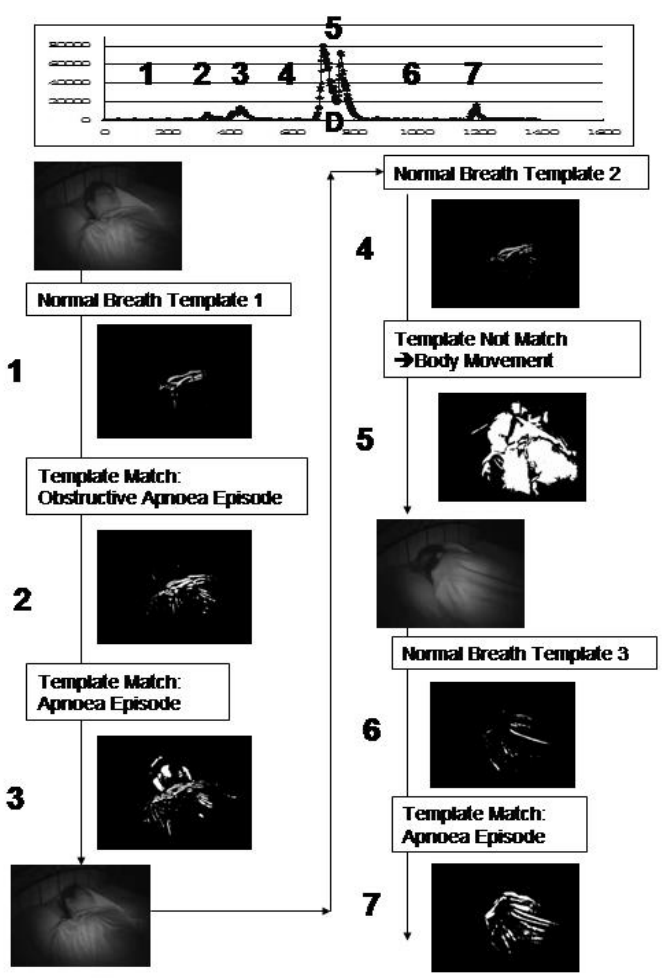

Fig. 5 Analysis Results

D. System Flow

Fig. 6 illustrates the system flow of the proposed approach. The proposed algorithm is displayed in section $\mathrm{G}$ in details. 


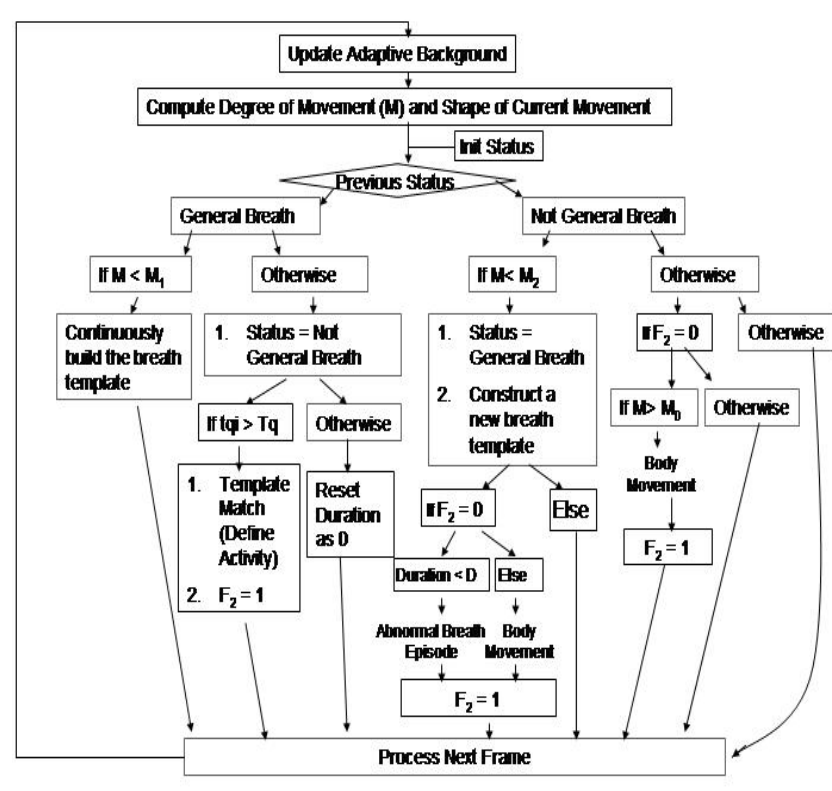

$\mathrm{M}_{1}$ : Threshold to change status from "general breath" to "not-general breath" $\mathrm{M}_{2}$ : Threshold to change status from "not-general breath" to "general breath"

Fig. 6 System Flow

\section{E. Breath Template Construction}

The template represents a combination of shapes of general breathing movements. It is continuously built over time, and the development does not stop until the status changes to "not general breath". Then the template will be used for breath recognition.

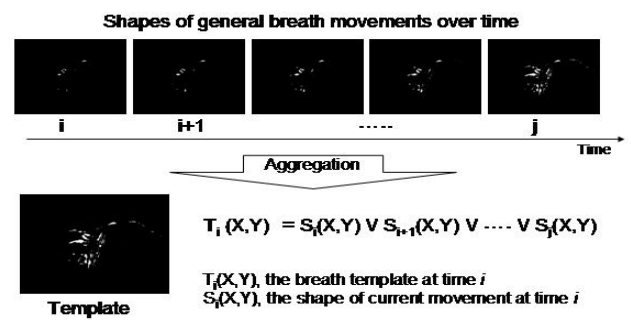

Fig. 7 Template Construction

A template quality index is created to decide whether the template is mature enough to use in matching the shape of current movement or not. Patients with shallow breathing patterns lead to a low quality general breath template. Alternatively, we use the degree of movement (M) and duration of the movement to eliminate the occurrences of body movement.

\section{F. Template Match}

The proposed method compares the breath template and the shape of the current movement. To classify the desired events, an index $E$ is calculated, as in the following equation, and used as demonstrated in figure 8 .

$$
\begin{aligned}
& E=W_{2} / W_{1} \\
& \text { Where: } \\
& W_{1}=T_{i}(x, y) \wedge S_{i}(x, y) ; W_{2}=\sim T_{i}(x, y) \wedge S_{i}(x, y) \\
& \wedge \text { is the AND operation; } \sim \text { is the negative of the image } T .
\end{aligned}
$$

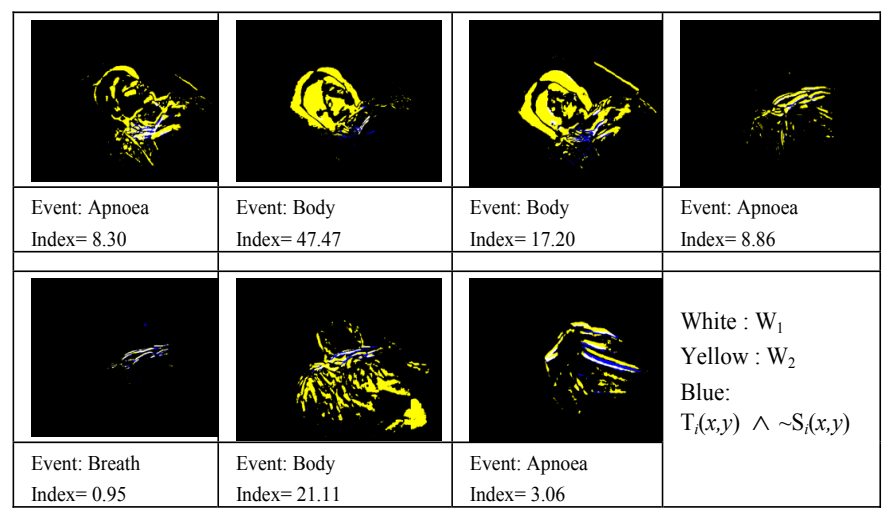

Fig. 8 Template Match Results

\section{G. Algorithm}

Inputs:

1. $\mathrm{P}_{i}(x, y)$, the intensity value at location $(x, y)$ of the image $\mathrm{P}$, at time $i$.

$x=\{1,2, \ldots, \mathrm{q}\}$; where $\mathrm{q}$ is the total number of columns of each frame/image

$y=\{1,2, \ldots, \mathrm{k}\}$; where $\mathrm{k}$ is the total number of rows in each frame/image $\mathrm{P}_{i}(x, y)=\{0,1, \ldots, 255\}$

2. $\mathrm{M}_{0}$ : The large motion threshold used to determine events as body movement

3. D: Threshold for time duration of the apnoea event

4. $\mathrm{T}_{\mathrm{Q}}$ : Threshold to use/not use the current template

5. $\mathrm{M}_{2}$ : Motion threshold to switch from "not general breath" to "general breath" state

6. N: Noise filtering threshold for each individual pixel $\mathrm{P}_{i}(x, y)$

7. $\mathrm{M}_{3}$ : Motion threshold for initializing $\mathrm{S}$ [step 4.1]

8. $\mathrm{nt}_{1}$ : Number of frames with low motion degree for initializing $\mathrm{S}$ [step 4.2]

9. $\mathrm{nt}_{2}$ : the number of the frame for template matching

10. $\mathrm{nt}_{3}$ : the number of the frame for updating $\mathrm{M}_{1}$

11. $t_{2}, t_{3}$ : threshold for template matching

\section{Output:}

Predicted result Ri, where R is ( 0: Apnoea, 1: Body Movement, 2: Breath), where $\mathrm{i}$ is time $\mathrm{i}$.

\section{Parameter:}

1. $\quad \mathrm{S}:(0:$ not general breath, 1 : general breath)

2. $\mathrm{F}_{1}:(0:$ template match On, $1:$ template match Off $)$

3. $\mathrm{F}_{2}:(0$ : define current event On, $1:$ define current event Off)

4. $\mathrm{M}_{1}$ : Motion threshold to switch from "general breath" to "not general breath" state

5. $\quad \mathrm{B}_{i}(\mathrm{X}, \mathrm{Y})$; the adaptive background at time $i$

6. $\quad \mathrm{T}_{i}(\mathrm{X}, \mathrm{Y})$, the breath template at time $i$

7. $\mathrm{S}_{i}(\mathrm{X}, \mathrm{Y})$, the shape of current movement at time $i$

8. M: the degree of current movements

9. $\mathrm{C}_{1}, \mathrm{C}_{2}, \mathrm{C}_{3}, \mathrm{C}_{4}, \mathrm{C}_{5}$ : counters

10. tqi: Template quality index (the number of data points) in the breath template

\section{Steps:}

For $\mathrm{i}=1$ to $\mathrm{m}$, where $\mathrm{m}$ is the total number of video frames, then

** Initialise background

1. $\mathrm{B}_{1}(\mathrm{X}, \mathrm{Y})=\mathrm{P}_{1}(\mathrm{X}, \mathrm{Y})$ and process next frame and start from step 2 .

** Update the background

2. if $\mathrm{i} \% 3=0$, then update the background (Adaptive Background Model)

2.1 For $\mathrm{x}=1$ to $\mathrm{q}$ and for $\mathrm{y}=1$ to $\mathrm{k}, \Delta \mathrm{I}(x, y)=\mathrm{P}_{i}(x, y)-\mathrm{B}_{i-1}(x, y)$

2.2 if $\Delta \mathrm{I}(x, y)>0$, then

2.2.1 $\quad$ if $(\Delta \mathrm{I}(x, y)<1)$, then $\mathrm{B}_{i}(x, y)=\mathrm{B}_{i-1}(x, y)+1$

2.3 Else if $\Delta \mathrm{I}(x, y)<0$, then

2.3.1 $\operatorname{if}(|\Delta \mathrm{I}(x, y)|<1)$, then $\mathrm{B}_{i}(x, y)=\mathrm{B}_{i-1}(x, y)-1$

2.3.2 Else, $\mathrm{B}_{i}(x, y)=\mathrm{B}_{i-1}(x, y)-\Delta \mathrm{I}(x, y)$

** Compute Degree of Movement and Shape of current movement

3. For $\mathrm{x}=1$ to $\mathrm{q}$ and for $\mathrm{y}=1$ to $\mathrm{k}$,

$3.1 \quad \Delta \mathrm{II}(x, y)=\mathrm{P}_{i}(x, y)-\mathrm{B}_{i}(x, y)$

3.2 if $(\Delta \mathrm{II}(x, y)>=\mathrm{N}), \mathrm{S}_{i}(x, y)=255$ and $\mathrm{M}=\mathrm{M}+1$. 
3.3 Else, $\mathrm{S}_{i}(x, y)=0$

** Initialize S: look for first "general breath" mode

4. if $\mathrm{S}$ is not initialized yet, then

4.1 if $\left(\mathrm{M}<\mathrm{M}_{3}\right), \mathrm{C}_{1}=\mathrm{C}_{1}+1$.

4.2 if $\left(\mathrm{C}_{1}>\mathrm{nt}_{1}\right), \mathrm{S}=1$. Else, process next frame.

** Do template match

5. if $\mathrm{F}_{1}=0$, then do template match.

$5.1 \mathrm{C}_{2}=\mathrm{C}_{2}+1$

$5.2 \mathrm{if}\left(\mathrm{C}_{2}>\mathrm{nt}_{2}\right)$, then (do template matching and define the event as follows) 5.2.1 for $\mathrm{x}=1$ to $\mathrm{q}$ and for $\mathrm{y}=1$ to $\mathrm{k}$, then

$$
\begin{array}{r}
\text { 5.2.1.1 } W_{1}=\sum_{x=1}^{q} \sum_{y=1}^{k} n(x, y) \\
\text {,where } n(x, y)=1 \text { if } \mathrm{T}_{\mathrm{i}}(x, y)>0 \wedge \mathrm{S}_{i}(x, y)>0 \\
\text { 5.2.1.2 } W_{2}=\sum_{x=1}^{q} \sum_{y=1}^{k} n(x, y) \\
\text {,where } n(x, y)=1 \text { if } \mathrm{T}_{\mathrm{i}}(x, y)=0 \wedge \mathrm{S}_{i}(x, y)>0
\end{array}
$$

5.2.1.3 if $\left(\mathrm{W}_{2} / \mathrm{W}_{1}>\mathrm{t} 2\right)$, then $\mathrm{Ri}=1$

5.2.1.4 else if $\left(\mathrm{W}_{2} / \mathrm{W}_{1}>\mathrm{t} 2\right)$, then $\mathrm{Ri}=0$

5.2.1.5 else, $\mathrm{Ri}=2$

$5.2 .2 \quad \mathrm{~F}_{1}=1$

5.2.3 $\quad \mathrm{F}_{2}=1$

5.2.4 $\quad \mathrm{C}_{2}=0$

5.2.5 Process next frame

5.3 Else, process next frame.

**if previous status is "general breath", check if need to change status or update the breath template

6. If $\mathrm{S}_{\mathrm{i}-1}=1$, then

6.1 If $\left(\mathrm{M}>\mathrm{M}_{1}\right)$, then

$$
\begin{array}{lll}
6.1 .1 & & \mathrm{~S}=0 \\
& 6.1 .2 & \text { If }\left(\text { tq }>>\mathrm{T}_{\mathrm{Q}}\right), \mathrm{F}_{1}=0 \\
6.1 .3 & \text { Else, } \mathrm{C}_{3}=0 \\
6.1 .4 & \text { Process next frame }
\end{array}
$$

6.2 Else, update breath template and define $\mathrm{M}_{1}$ based on the person's general breath motion degree.

6.2.1 for $\mathrm{x}=1$ to $\mathrm{q}$ and for $\mathrm{y}=1$ to $\mathrm{k}$, then

6.2.1.1 if $\mathrm{T}_{i}(x, y)=0 \wedge \mathrm{S}_{i}(x, y)>0$, then $\mathrm{T}_{i}(x$,

6.2.2 $\mathrm{C}_{4}=\mathrm{C}_{4}+1$

$y)=255$ and tqi $=$ tqi +1

6.2.3 if $\left(\mathrm{C}_{4}=\mathrm{nt}_{3}\right), \mathrm{M}_{1}=$ tqi $* 2.2$.

6.2.3.1 if $\left(M_{1}<400\right), M_{1}=400$

**if previous status is "not general breath", check if need to change status or define the event

7. Else, then

7.1 $\mathrm{C}_{3}=\mathrm{C}_{3}+1$

7.2 if $\left(F_{2}=0\right.$ and $\left.M>M_{0}\right)$, then $\mathrm{Ri}=1$ and $F_{2}=1$

7.3 Else if $\left(\mathrm{M}<\mathrm{M}_{2}\right)$, then

7.3.1 $\quad \mathrm{C}_{5}=\mathrm{C}_{5}+1$

7.3.2 if $\left(\mathrm{F}_{2}=0\right)$, then define the event

7.3.2.1 $\mathrm{F}_{2}=1$

7.3.2.2 if $\left(\mathrm{C}_{3}>\mathrm{D}\right), \mathrm{Ri}=1$

7.3.2.3 Else, $\mathrm{Ri}=0$

7.3.3 $\quad$ If $\left(\mathrm{C}_{5}>10\right)$, then

7.3.3.1 $\mathrm{S}=1$

7.3.3.2 for $\mathrm{x}=1$ to $\mathrm{q}$ and for $\mathrm{y}=1$ to $\mathrm{k}, \mathrm{T}_{i}(x, y)=0$

7.3.3.3 tqi $=0$

7.3.3.4 $\mathrm{C}_{4}=0$

7.3.3.5 $\mathrm{F}_{2}=0$

7.3.3.6 $\mathrm{C}_{5}=0$

7.4 Process next frame

\section{EXPERIMENTAL SETUP}

Two SONY infrared camcorders (DCR-HC-30E) are utilized to capture 10 video clips from three different shooting angles. The infrared video frames were acquired at 15 frames per second, with resolution of $320 * 240$. The video and audio data is first captured with the WMP9 compression algorithm to minimize storage size, and then decompressed for off-line analysis. Moreover, in order to simulate the environment for diagnosis on sleeping disorders, there was no visible lighting in the filming room and the subjects were partially covered by a sheet. Furthermore, the experimental data was collected from two subjects with mainly 3 different postures (i.e. lying on the back, sleeping on one side and facing the camera, sleeping on the other side with their back facing the camera). The data was also collected in different days, with the subjects wearing different clothing. Activities, such as general breath, obstructive apnoea and body movement, were simulated by the subjects.

\section{EXPERIMENTAL RESULTS}

The proposed breathing activity recognition technique is used to identify abnormal breathing events and to distinguish abnormal breathing events from general body movements. Fig. 9 shows the calculated degree of motion, over time, for each video clip and Fig. 10 presents real events and analyzed results, which demonstrates the proposed approach achieves high accuracy in detecting abnormal breathing activity. The real events are defined by human observation from the video content.

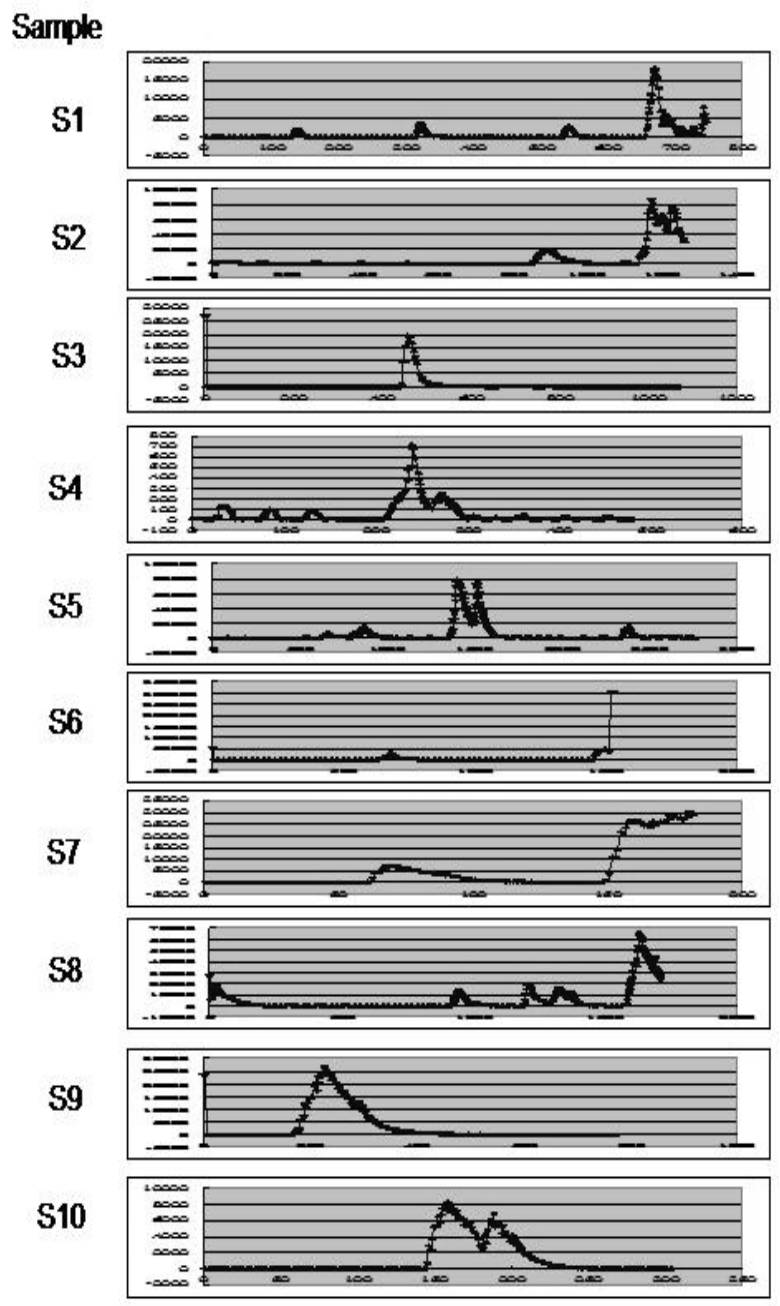

Fig. 9 Experimental Results - Motion Degree 


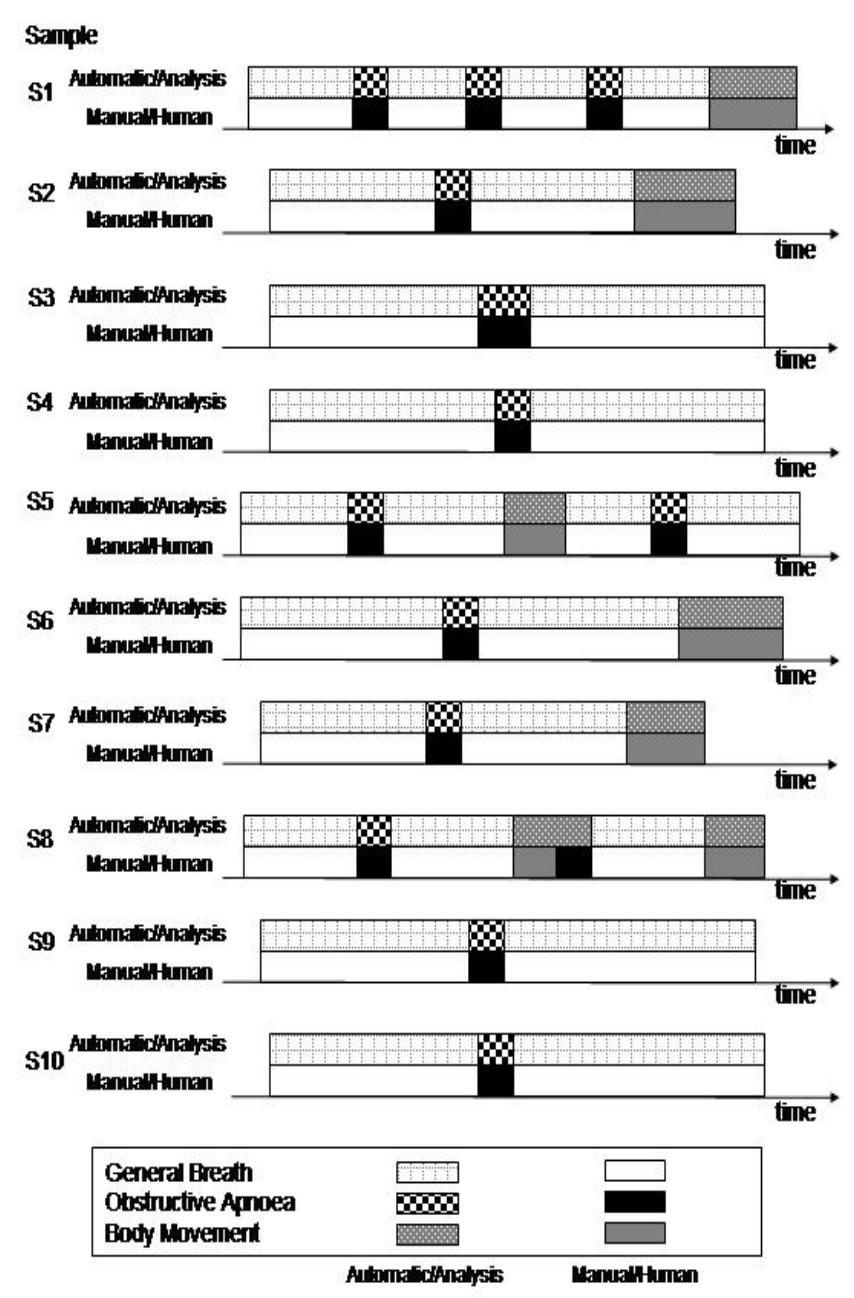

Fig. 10 Experimental Results - Analysis and Real Events

\section{CONCLUSION}

In this paper, we presented a non-invasive approach for recognizing abnormal breathing activity in assisting diagnosis of obstructive sleep apnoea. The proposed approach utilizes infrared video information and avoids imposing geometric constraints or position limitations. The method is validated with limited experiments simulating the real environment for diagnosis of sleep disorders. Although the presented approach is mainly developed for diagnosis of obstructive sleep apnoea, we believe that it can be utilized in other medical applications that require monitoring of the breath activity.

\section{FUTURE WORK}

We are investigating more automatic ways for obtaining the threshold values and adapt them to individual subjects, to accommodate human variations. We are also planning to support the video analysis of the human breath activity by adding audio analysis.

\section{ACKNOWLEDGMENTS}

We would like to thank the United Lincolnshire Hospital NHS Trust for supporting the PhD scholarship of C.W. Wang. We thank Dr. Neil Gravill and Dr. Simon Matusiewicz for their valuable comments in obstructive sleep apnoea.

\section{REFERENCES}

[1] Hyun Medics, Baby breath monitor, http://hyun.en.ecplaza.net/14.asp

[2] Svetlana I., Mammo H. Y., John W. A., Michael E. H., Carl H. M., and Scott R. P., "A gated deep inspiration breath-hold radiation therapy technique using a linear position transducer," Applied Clinical Medical Physics 6(1), Winter 2005

[3] David W. G., "Heart-respiratory monitor - infants", http://www.nlm.nih.gov/medlineplus/ency/article/007236.htm, Copyright 2005, A.D.A.M., Inc

[4] George B. M., Roger G. M., Marjorie A. B., Joseph S. W., Aaron D. B., Joseph E. M., and Ary L. G., "Clinical Validation of the ECG-Derived Respiration (EDR) Technique," Computers in Cardiology 13:507-510, 1986

[5] Amin H. M., Cigada M., Fordyce W. E., and Camporesi E. M., "Noninvasive monitoring of respiratory volume. Experimental evaluation of a breath monitoring device," Anaesthesia.48(7):608-10, July 1993

[6] David C. M., Steve W. K., Majd A., Beverely T., and Robin A. F., "Noninvasive analysis of physiological signals (NAPS): a vibration sensor that passively detects heart and respiration rates as part of a sensor suite for medical monitoring," Summer Bioengineering conference, Florida, 2003

[7] Randall D. P., "Remote Respiratory Monitor," Proceedings of the $8^{\text {th }}$ Annual IEEE Symposium on Computer-Based Medical Systems, 1995

[8] Flemons W. W., Littner M.R., Rowley J. A., Gay P., Anderson W. M., Hudgel D. W., McEvoy R.D., and Loube D.I., "Home Diagnosis of Sleep Apnea: A Systematic Review of the Literature: An Evidence Review," Chest 124:1543-1579, 2003

[9] Storck K., Karlsson M., Ask P., and Loyd D., "Heat Transfer Evaluation of the Nasal Thermistor Technique," IEEE Transactions on Biomedical Engineering 43(12):1187-1191, December 1996

[10]Murthy R., Pavlids I., and Tsiamyrtzis P., "Touchless Monitoring of Breathing Function," Proceeding of the $26^{\text {th }}$ Annual International Conference of the IEEE EMBS, 2004.

[11]Zhu Z., Fei J., and Pavlidis I., "Tracking Human Breath in Infrared Imaging," Proceeding of the $5^{\text {th }}$ IEEE Symposium on Bioinformatics and Bioengineering, 2005.

[12]Matusiewicz S. [Position: Consultant Physician], Personal Interview, Medical Physics Department of Lincoln County Hospital in United Kingdom, 2006 